\title{
Proyecto: Construyamos centros educativos inclusivos. Un modelo para evaluar actitudes, políticas y acciones institucionales
}

\section{María Gabriela Marín Arias* Lady Meléndez Rodríguez**}

\author{
*Encargada de la Cátedra de Educación Especial. Universidad Estatal a Distancia (UNED), Costa Rica; mmarin@uned.ac.cr \\ **Encargada del Programa de Educación Especial. Universidad Estatal a Distancia (UNED), Costa Rica; Imelendez@uned.ac.cr
}

Recibido: 6 de junio de 2011

\section{RESUMEN}

El proyecto "Construyamos centros educativos inclusivos" pretende coadyuvar en la transición de las instituciones educativas hacia la educación inclusiva, mediante el análisis de las actitudes, políticas y acciones institucionales. Para el logro de dicho propósito se han desarrollado actividades en tres centros de educación primaria: Escuela José Figueres Ferrer, Escuela Granadilla Norte y Escuela Monterrey Vargas Araya, que hoy constituyen centros modelo de educación inclusiva. La Universidad Estatal a Distancia, mediante el Programa de Educación Especial, inició el proyecto en el año 2008, con el patrocinio de la Agencia Española de Cooperación Internacional para el Desarrollo (AECID) y en coordinación con el Instituto Universitario de Integración en la Comunidad (INICO) de la Universidad de Salamanca y la Facultad de Educación de la Universidad de Costa Rica. A lo largo del proceso fue preciso acercarse a otras instancias como la Comisión Nacional de Educación Inclusiva y el Centro Nacional de Recursos para la Educación Inclusiva (CENAREC), ambos pertenecientes al Ministerio de Educación Pública (MEP); entidad que hoy intenta replicar el modelo surgido de este proyecto en otros centros educativos del país.

\section{PALABRAS CLAVE}

Inclusión, educación inclusiva, atención a la diversidad, centros educativos inclusivos, autoevaluación, proyecto de centro.
Aceptado: 18 de octubre de 2011

\section{SUMMARY}

The "Construyamos centros educativos inclusivos" project, seeks to assist in the transition of schools towards inclusive education, by analyzing the attitudes, policies and institutional actions. To achieve this objective, actions have been developed in three schools of Primary education: Escuela José Figueres Ferrer, Escuela Granadilla Norte and Escuela Monterrey Vargas Araya, which today are models of inclusive education. The Universidad Estatal a Distancia, through the Special Education Program, started the project in 2008, sponsored by the Agencia Española de Cooperación Internacional para el Desarrollo (AECI) in coordination with the Instituto Universitario de Integración en la Comunidad (INICO) of the Universidad de Salamanca and the Facultad de Educación at the Universidad de Costa Rica. Throughout the process, the need to look for other instances that would support the project, was identified, which is why partnerships were established with agencies such as the Comisión Nacional de Educación Inclusiva and the Centro Nacional de Recursos para la Educación Inclusiva (CENAREC), both belonging to Ministerio de Educación (MEP), entity which tries to replay the model, product of this project, in others Costa Rican schools.

\section{KEY WORDS}

Inclusion, inclusive education, attention to diversity, inclusive schools, self-evaluation, the School Project. 


\section{Justificación}

La Declaración de Educación para Todos por la United Nations Education Science and Culture Organization (UNESCO), emitida en el año 1990 y evaluada en Dakar en el 2000, ha señalado incansablemente la necesidad de que cada país y región desarrolle estrategias para la atención educativa de todas las personas, especialmente a aquellas cuya condición de desventaja o vulnerabilidad las coloca en riesgo de quedar excluidas de los beneficios de la educación.

No obstante, la clara argumentación de la UNESCO en apoyo a la Educación para Todos no ha sido suficiente para que esta se cumpla, sobre todo en la región Centroamericana. Una de las razones de su incumplimiento se achaca a la falta de orientaciones e instrumentos metodológicos, que permitan ejecutar estrategias pertinentes para la inclusión educativa en todos los sectores y niveles de la educación.

El Informe del Estado de la Educación del 2005 en Costa Rica advierte que el abandono escolar debe considerarse como un proceso de exclusión del sistema, cuyas formas de educación resultan muy poco atractivas, sobre todo para muchos jóvenes de la educación secundaria. Encuestas realizadas entre desertores revelan que la mayoría no puede precisar el aporte que recibieron en la escuela, ni saben qué les hubiera gustado obtener mientras permanecieron en el sistema.

Está claro que no son las condiciones socioeconómicas las únicas ni, quizás, las más importantes para provocar la exclusión educativa, sino la grave falta de pertinencia curricular y de participación de los educadores y de otros actores del proceso educativo en la definición, diseño y aplicación de un currículo de calidad y con pertinencia para todos sus participantes.

Por las razones anteriores es posible juzgar que las medidas de compensación socioeconómica son totalmente insuficientes e ineficientes para mejorar y equiparar las condiciones en las que se aprende y se enseña en este país (Meléndez, 2006) y en el resto de la región. Como bien argumenta Hoyos "Un Estado que no cumple el principio fundamental de equidad facilitando educación de calidad a todos los ciudadanos carece de legitimidad para promover y defender la convivencia y para el fomento y administración del bien común" (2003, p.14). Por lo que nos atrevemos a decir que un país con las pretensiones de desarrollo educativo que tiene el nuestro, debe, ante todo, apostarle a la equidad con calidad, aplicando todos los medios posibles para lograr una educación inclusiva en todos los centros educativos.

Por tal motivo, el proyecto que enseguida se detalla se propuso caminar hacia la inclusión educativa y evitar la exclusión, a partir de prácticas que se generen desde el corazón mismo de los centros educativos. Nuestro trabajo busca construir centros educativos inclusivos con base en un análisis de las actitudes, políticas y acciones institucionales, el fortalecimiento de aquellas que favorecen el trato equitativo y de calidad de todos los alumnos y la evitación de otras que más bien promueven la discriminación y la exclusión de los niños más vulnerables, especialmente de aquellos que presentan necesidades educativas especiales. Asimismo, guarda la intención de constituirse en un modelo digno de imitar por otros centros escolares en este país y en el resto del territorio centroamericano.

\section{- Fundamentación}

En promedio, cerca del $40 \%$ de los habitantes de la región latinoamericana son pobres $y$, de estos, aproximadamente 81 millones viven en pobreza extrema o indigencia, lo que implica que no son capaces de atender más del $65 \%$ de sus necesidades básicas. El resto, casi $120 \mathrm{mi}-$ llones de personas, se encuentra en el espacio denominado pobreza relativa o en condición de vulnerabilidad.

En el caso de los países de Centroamérica, las cifras promedio de pobreza son mayores $(46,5 \%$ 
de pobreza general y $19,7 \%$ de pobreza extrema), entre los que destaca Honduras, donde el $67,8 \%$ de la población se considera en pobreza general, con un lamentable $48,1 \%$ de pobreza extrema. Le siguen Guatemala con $51 \%$ y Nicaragua con $48,3 \%$ de habitantes en condición general de pobreza. (Programa Estado de la Nación, 2008). tenido acceso a los ambientes de aprendizaje, más aquellos que no cuentan con los recursos para seguir adelante, no es difícil entender las razones que hacen que el aumento de los círculos de pobreza en proporción al crecimiento poblacional, se acentúe (Programa Estado de la Nación, 2003).

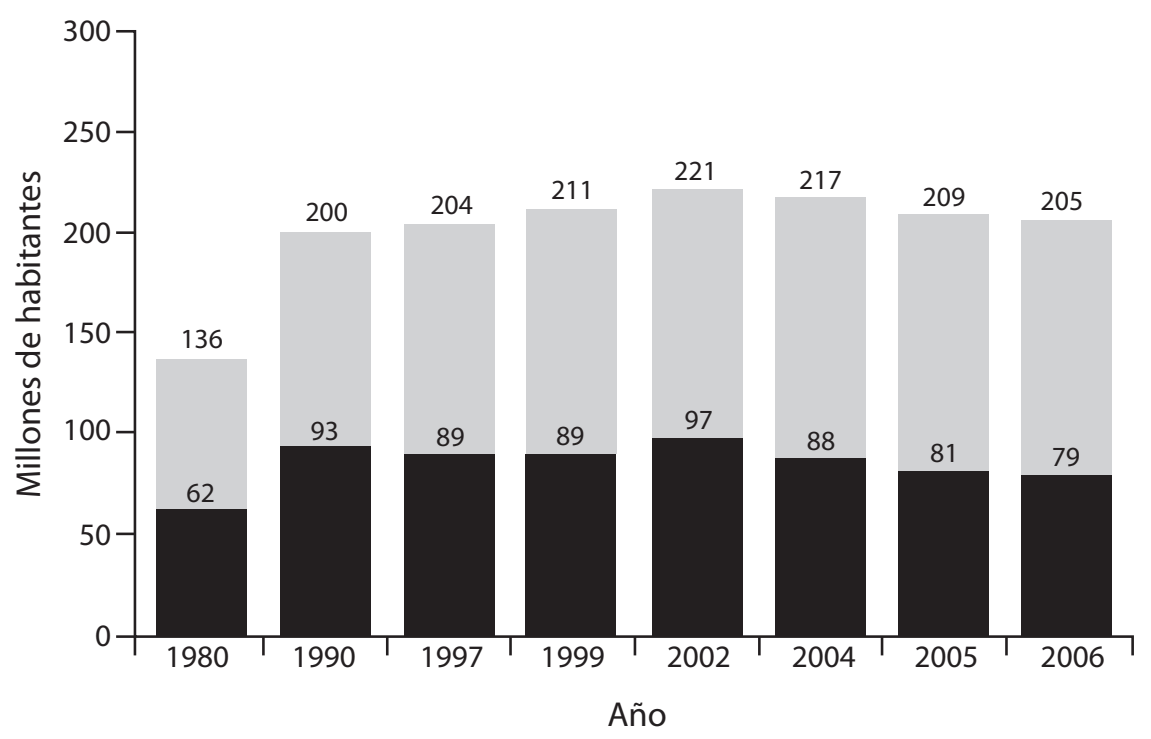

América Latina: Pobreza e indigencia.

Fuente: CEPAL, 2006.

El desarrollo se logra con equidad y con oportunidades de vida con calidad para todos los habitantes de una nación. Por esa razón, el esfuerzo por la inclusión es un esfuerzo por la equidad, el desarrollo y los derechos humanos. Toda acción selectiva con respecto a las oportunidades de desarrollo deriva en alguna forma de retroceso, pobreza y exclusión social, pero esto ha sido muy difícil de entender para los gobiernos de nuestros países.

Con respecto a la educación, por ejemplo, se suma que la obsesión por la norma y el desprecio a las diferencias han hecho que la escuela empuje a muchos de sus alumnos hacia la exclusión de las oportunidades de educarse. Si a estos menores sumamos a quienes no han
En síntesis, hasta ahora hemos intentado explicar que la problemática planteada por la exclusión en América Latina, determinada principalmente por sus condiciones de pobreza y de fragmentación social, es compleja, profunda y perpetuada por la exclusión educativa. Lo anterior da pie para inducir que cualquier esfuerzo que se haga por revertirla será un punto a favor para la inclusión social y el desarrollo de la región. Por lo que vale la pena poner atención sobre las buenas prácticas inclusivas, sin conformarse, por supuesto, con experiencias aisladas al respecto, en tanto esta situación constituye un agudo y enmarañado problema estructural que, como tal, debe ser atendido a fin de alcanzar una verdadera transformación social. 
En ese sentido, el Informe Mundial Mejor Educación para Todos: Cuando se nos incluya también (Inclusión Internacional e INICO, 2009, pp. 156-157), expone que:

a) Existe un vacío político de liderazgo y responsabilidad para la Educación Inclusiva, en cuya carencia no se reformarán los sistemas educativos.

b) Las familias no reciben apoyo para tener altas expectativas para sus hijos con discapacidad, y la educación inclusiva para la mayoría de ellos permanece fuera de la imaginación. En consecuencia, la demanda efectiva es mínima.

c) En su mayor parte, los maestros carecen de la formación, el liderazgo, los conocimientos y los apoyos para adaptar el plan de estudios y hacer que las aulas inclusivas funcionen.

d) Las personas que necesitan los conocimientos sobre la Política inclusiva y la práctica para trabajar -los padres, los maestros, los administradores y los encargados de formular políticas no están eficazmente enlazados desde el plano local con las redes de conocimientos mundiales para la Educación Inclusiva.

e) La "maquinaria" de un sistema de Educación Inclusiva no ha sido establecida en la mayoría de los casos; es decir, la gestión, la política, la planificación, la financiación, la aplicación y la supervisión necesarias.

f) Finalmente, el público en general parece estar atrapado en una "solidaridad de negación" sobre la realidad de la exclusión (...) La falta de apoyo público para la inclusión es una de las mayores barreras a enfrentar. Perpetúa los estereotipos negativos y socava el liderazgo político.

Con respecto a lo que se entiende por inclusión educativa este proyecto acoge la definición de
Arnaiz (2005). Dicha definición explica que la inclusión educativa se opone a cualquier forma de segregación, a cualquier argumento que justifique la separación, a cualquier pretexto en el ejercicio de los derechos a la educación. La inclusión es un planteamiento comprometido que refleja la defensa de unos valores determinados que se relacionan con los fines de la educación, con la forma de sociedad que queremos vivir (Arnaiz, 2005), por lo cual la educación tiene como objetivo fundamental permitir que cada ciudadano reciba el acceso al conocimiento de los productos propios de su cultura.

Es necesario destacar el hecho de que aún cuando los distintos actores de la educación parecen estar de acuerdo con las demandas de la inclusión, se ha desarrollado una serie de reflexiones al respecto, pero pocas orientaciones prácticas para poder llevarla a cabo. Entre éstas destaca el manual de indicadores desarrollado por Anthony Booth y Mell Ainscow en el Reino Unido.

Con la intención de colaborar con los centros educativos en su deseo de ser cada vez más inclusivos, Booth y Ainscow (2000) diseñaron una herramienta que les permitiera autoevaluarse desde esa perspectiva, además de definir políticas y proyectos que los guiara hacia el cumplimiento de ese deseo. Dicha herramienta, en su versión castellana, se titula "Índice de Inclusión: desarrollando el aprendizaje y la participación en las escuelas" y empieza a ser utilizada con muy buen suceso en muchos centros escolares del mundo, por lo que la presente propuesta se fundamenta, precisamente, en el uso adaptado para Costa Rica de este mismo manual.

Según Essomba (2003), una escuela que invita a la participación y que dentro de su estructura organizativa puede atender a la diversidad es una escuela para diferentes etnias, idiomas, géneros, costumbres, estilos de aprendizaje, dudas, intereses e inquietudes. Además, una escuela inclusiva debe procurar atender las necesidades de cada uno de los miembros que 
componen la comunidad escolar, sean estos padres, estudiantes, profesorado, funcionarios administrativos y miembros de la comunidad en la que está inserta la escuela.

Por lo tanto, como explican Booth y Ainscow, (2000) una escuela con intenciones de ser inclusiva debe replantear y transformar su quehacer, abrirse a nuevas oportunidades y proyectos, además de fusionarse con su comunidad.

\section{- Definición del problema}

Los principales problemas de la inequidad y exclusión en las oportunidades de acceso a una educación de calidad en nuestro país apuntan hacia la falta de atención educativa a la diversidad, dejando excluidos de la educación a:

a) Los que no han tenido y no tienen medios para llegar al centro educativo (porque ese centro no existe o porque la brecha espacial y económica no se los permite).

b) Los que llegan, pero reciben un trato en desventaja por razones personales y culturales (población indígena, rural marginal, urbana marginal, migrante en situación de pobreza, con necesidades educativas especiales entre otros).

c) Los que asumen el fracaso escolar como propio y huyen del sistema (dificultades de permanencia).

En función de los puntos anteriores, este proyecto tiene como finalidad contribuir al mejoramiento de las prácticas escolares, mediante la puesta en práctica de una metodología participativa e inclusiva, y con ello contribuir, además, a la construcción de planes de mejoramiento orientados a detener la exclusión desde los mismos centros educativos.

La investigación planteada involucra la ejecución de procesos de autoevaluación por parte de los centros escolares, con el fin de que ellos mismos identificaran los aspectos positivos y negativos que se derivan de su quehacer en cuanto a la vivencia o ausencia de la inclusión educativa. Así, a partir del proceso de autoevaluación cada uno diseñaría un plan que le permitiera superar las debilidades detectadas y elevar su calidad a partir de la condición cada vez más inclusiva de sus servicios.

\section{Objetivos}

Para su cumplimiento, el proyecto se propuso alcanzar los objetivos que enseguida se exponen.

\section{Objetivo general}

Favorecer la inclusión y evitar la exclusión educativa mediante la transición de las escuelas hacia centros educativos inclusivos.

\section{Objetivos específicos}

i. Promover la autoevaluación del quehacer escolar desde el enfoque de la educación inclusiva.

ii. Facilitar procesos de autoevaluación, construcción de políticas y planificación de acciones inclusivas en los centros educativos participantes.

iii. Sistematizar el proceso de construcción de centros educativos inclusivos.

\section{METODOLOGÍA}

Este trabajo se propuso desde la investigaciónacción, e incluye cinco importantes grupos de acciones: a) capacitación a los comités coordinadores y funcionarios de los centros participantes, b) autoevaluación de los centros, c) sistematización del proceso de autoevaluación por parte de los comités coordinadores, d) diseño de los proyectos de centro, e) observación, registro y sistematización de todo el proceso vivido por los centros por parte de las investigadoras. 


\section{Metodología de la capacitación}

Se utilizó como guía fundamental el Index for Inclusion (2000) de los autores Mel Ainscow y Tony Booth, en su versión traducida por OREALC-UNESCO para América Latina y adaptada para Costa Rica por el Equipo de Docentes de Apoyo del circuito escolar 10 de la Región Educativa de San José (2008); la que permitió organizar y desarrollar charlas, documentos y actividades de aprendizaje colaborativo para los participantes.

\section{- Metodología de la investigación}

Se siguió la metodología de investigaciónacción propuesta por Alguacil, et al. (2006) y Corbetta (2007), donde las investigadoras y su asistente participaron como facilitadoras de los procesos, recopiladoras, analistas y sistematizadoras de la información.

Se consideraron participantes de la investigación a todos los miembros del personal docente y administrativo comprometidos con las acciones de autoevaluación y diseño del proyecto educativo institucional, a las facilitadoras involucradas, estudiantes, padres de familia, miembros del patronato y junta escolar y otras personas de la comunidad.

Se registró información mediante una bitácora de campo a partir de la revisión de documentos de los centros y de minutas de los comités coordinadores, se creó un archivo fotográfico y fílmico, posteriormente se compiló y analizó el material utilizado en cada sesión de trabajo.

Se sistematizó la información y se redactó un reporte final del proceso, siguiendo las categorías de análisis dictadas por la misma dinámica de acción.

Se documentó el consentimiento informado de los participantes, tanto para ser incluidos en la capacitación, como en su calidad de informan- tes y autoevaluadores. Además, se confirmó y validó la información mediante el uso de grupos focales y registros de discusión plenaria de los participantes.

Por su lado, los participantes asistieron a los talleres de capacitación, aplicaron los instrumentos de autoevaluación, desarrollaron la sistematización de su propio proceso y han avanzado en el diseño del proyecto de centro y la puesta en práctica de acciones concretas hacia la educación inclusiva.

\section{LOGROS Y ALCANCES}

En una etapa previa a la implementación del proyecto, en coordinación con la Universidad de Costa Rica y la Universidad de Salamanca se realizaron las siguientes actividades conjuntas:

»Estudio y análisis de la Guía para la evaluación y mejora de la educación inclusiva. Índex para la Inclusión (Booth y Ainscow, 2000)

» Establecimiento de descriptores para la conformación de un marco teórico de referencia sobre educación inclusiva y su marco legal en cada uno de los países participantes del proyecto: España y Costa Rica

»Construcción bibliométrica

$»$ Determinación de indicadores sobre educación inclusiva

»Desarrollo de capítulos teóricos con base en los indicadores, cada uno a cargo de una miembro del equipo. Lo anterior dio origen al libro "Aspectos clave de la educación inclusiva", publicado por el INICO de la Universidad de Salamanca

" Pasantía en Costa Rica de las investigadoras españolas y de las costarricenses en el País Vasco y Salamanca para observar experiencias de educación 
inclusiva y recopilar material pertinente para el proyecto

En el marco del desarrollo del proyecto, los centros educativos participantes, en coordinación con el personal a cargo del proyecto en la Universidad Estatal a Distancia, han realizado las siguientes acciones:

Reuniones de coordinación de los miembros integrantes de los comités de las escuelas participantes, con personal de la Universidad Estatal a Distancia (UNED), con el propósito de brindar capacitación continua, definir políticas y lineamientos de trabajo, compartir experiencias y coordinar el trabajo a seguir

»Coordinación con la Comisión Nacional de Educación Inclusiva y el Centro $\mathrm{Na}$ cional de Recursos para la Educación Inclusiva con el fin de capacitar al personal de las escuelas, en el diseño del Proyecto Institucional de Centro y la sistematización del informe del proyecto respectivamente, con la intención de que las acciones estuvieran acordes en todo momento con las disposiciones ministeriales y que no implicaran esfuerzos adicionales para los docentes ni directores de los centros
» Capacitaciones al personal docente y administrativo de los centros educativos participantes

»Adaptación de instrumentos para la autoevaluación

»Revisión y tabulación de los datos de los cuestionarios

»Elaboración de la sistematización por parte de los centros educativos y valoración de las fortalezas y las debilidades de su proceso

»Elaboración de informes por parte del equipo investigador

» Desde su propia iniciativa y con apoyo de la UNED, los centros participantes han realizado las siguientes acciones:

» Capacitaciones sobre la atención educativa a la diversidad del alumnado y trabajo en equipo, cuyo fin primordial es prepararse para la construcción de nuevas políticas y ejecución de prácticas inclusivas

» Visitas a la comunidad para conocer la realidad del entorno que les rodea y preparase como centro educativo, para realizar una tarea que responda a las demandas sociales

»Aplicación de instrumentos de autoevaluación

"Disponerse para la elaboración del proyecto de centro, con base en las prioridades y fortalezas que han sido reveladas en los resultados de la autoevaluación.

En el momento de realizar este artículo quedan pendientes dos acciones importantes, como son el diseño de los proyectos institucionales desde un enfoque inclusivo para cada centro, así como la divulgación de esta experiencia mediante una publicación de buenas 
prácticas educativas y organizacionales, que dé a conocer los logros alcanzados en esta línea por cada uno de los centros participantes. En forma posterior a estas acciones, las investigadoras procederán a redactar el informe final y a realizar la devolución respectiva a los centros participantes.

\section{- Principales conclusiones}

1. Durante la fase de capacitación fue posible evidenciar que el autoconocimiento institucional de las condiciones y niveles de inclusión educativa debe surgir, sobre todo, de la autorreflexión de los miembros de la comunidad escolar. Por lo que es pertinente que los agentes externos se involucren solo mediando e induciendo los procesos, pero sin señalar en forma directa los aspectos que consideran que deben ser modificados para alcanzar las metas propuestas.

2. Después de haber pasado por el proceso de autoevaluación, los centros reconocen que:

a. Es posible alcanzar la educación inclusiva siguiendo un proyecto institucional, fundamentado en un diagnóstico que permita identificar hacia dónde se debe ir, cómo y con qué recursos.

b. El proyecto constituye un apoyo que colabora no sólo con la inclusión educativa de los alumnos, sino también con el trabajo que se achaca a sus procesos; por lo que es acogido positivamente por los miembros de la comunidad escolar.

c. Un proyecto de educación inclusiva está en posibilidad de mejorar no sólo las condiciones educativas de todos los estudiantes, sino los conflictos internos causados por diferencias culturales y carestías económicas, así como acercar e involucrar a las familias en las intenciones transformadoras del centro escolar, que pretenden además impactar positivamente a la comunidad.

d. Requieren la colaboración de entidades comunales y expertas en inclusión educativa así como en enfoques y en formas de investigación, como es el caso de las universidades involucradas, para sacar adelante su proyecto.

e. La principal debilidad es la falta de tiempo disponible para ejecutar todas las tareas que demanda el proyecto, por lo que es necesario solicitar a las autoridades escolares, la posibilidad de establecer una reorganización de la gestión que permita dedicar mayor tiempo a esta iniciativa.

3. Para las investigadoras es necesario tener presente en todo momento que la inclusión educativa no se trata de dar indicaciones desde sus propios criterios, ni de la provisión de recursos a los centros para mejorar sus condiciones, sino más bien de mediar e inducir los procesos para que los recursos que se tienen o se puedan obtener beneficien a todos los estudiantes con equidad y calidad.

4. De acuerdo con la experiencia llevada a cabo, las investigadoras determinan que:

a. El avance del proyecto en cada uno de los centros depende en gran medida de su organización, cultura y liderazgo institucional. También influye de forma significativa el apoyo del director (a) y las actividades propias del circuito escolar.

b. Es importante identificar una forma que permita que los directores 
de las escuelas que forman parte del proyecto puedan hacer pasantías internacionales en centros modelo, a la vez de divulgar su propia experiencia.

c. El recurso humano con que cuenta el proyecto es insuficiente para poder cumplir con sus demandas, por lo que se requiere de al menos dos medios tiempos de asistencia para cumplir oportunamente con cada fase.

d. Se recomienda que la UNED permanezca apoyando a los centros educativos participantes, con la finalidad de dar continuidad al proceso de puesta en práctica de los proyectos de las escuelas, sistematización de procesos y elaboración de informes de resultados.

\section{BIBLIOGRAFÍA}

Alguacil, J. Basagoiti, M. Bru, P y Camacho, J. (2006). Investigación- Acción Participativa en el Barrio de San Cristóbal de los Ángeles (Distrito de Villa Verde, Madrid). Cuadernos de Trabajo Social. 19, 331 - 346.

Arnaiz, P. (2005). Atención a la Diversidad. Programación curricular. Costa Rica: Editorial Universidad Estatal a Distancia.

Booth, T. y Ainscow, M. (2000). Índice de Inclusión Educativa. (Trad. Y Ed. OREALC/UNESCO). Chile: OREALC.

CEPAL (2006). Panorama social de América Latina. Extraído el 28 de marzo de 2009 desde http:// www.eclac.org/cgi-bin/getProd.asp?xml=/publicaciones/xml/

Corbetta, P. (2007). Teoría de la investigación Social. Métodos y Técnicas. Londres: SAGE Publications.

Estado de la Región (2003). Segundo Informe sobre Desarrollo Humano en Centroamérica y Panamá. Extraído el 28 de marzo de 2009 desde http://www.estadonacion.or.cr/Region2003/ Paginas/carpeta.html
Essomba, M. A. (coord.) (2003). Educación e inclusión social de inmigrados y minorías. Tejer redes de sentido compartido. España: Praxis.

Hoyos, G.(2003). Participación del Estado, de la Comunidad Académica y de la Sociedad en el Mejoramiento de la Calidad de la Educación Superior. Extraído el 28 de julio de 2006 desde http:// www.gov.co

Inclusion International/INICO (Octubre, 2009). Informe Mundial Mejor Educación para Todos: cuando se nos incluya también. Salamanca: INICO.

INEC. (2011). Costa Rica tiene 4301172 habitantes. Extraído el 28 de julio de 2006 desde http:// www.inec.go.cr

Meléndez, L. (2006). El Docente de Apoyo Educativo de cara a las Políticas de Inclusión Social en América Latina. 3er. Congreso Internacional de Discapacidad. Inclusión: oportunidades para todos. Memorias. Medellín, Del 20 al 23 de Septiembre de 2006.

MEP (2009). Boletín 05-9. Extraído el 28 de julio de 2006 desde http://www.mep.go.cr

Programa Estado de la Nación (2005). Primer Informe Estado de la Educación. Costa Rica: Estado de la Nación.

Programa Estado de la Nación (2008). Estado de la Región en Desarrollo Humano Sostenible. Un informe desde Centroamérica y para Centroamérica. Costa Rica: Estado de la Nación.

UNESCO (1990). Declaración Mundial de Educación para Todos. Marco de Acción Asociado. Tailandia: UNESCO.

UNESCO (2000). Marco de Acción de Dakar Educación para Todos: Cumplir con los Compromisos Comunes. Costa Rica: UNESCO/MEP: Proyecto SIMED. 
